\title{
Effective solution of families of Thue equations containing several parameters
}

\author{
by \\ Clemens Heuberger and Robert F. Tichy (Graz)
}

Dedicated to Professor Kálmán Györy on the occasion of his 60th birthday

1. Introduction. By his classical approximation theorem, A. Thue [27] proved that a Diophantine equation

$$
F(X, Y)=m,
$$

where $F \in \mathbb{Z}[X, Y]$ is an irreducible form of degree $n \geq 3$ and $m \neq 0$ a fixed integer, has only finitely many solutions. However, this proof is non-effective and does not give any bounds for the size of the possible solutions. In 1968, A. Baker could give effective bounds based on his famous theory on linear forms in logarithms of algebraic numbers. In the last decades, this method was refined (see for instance Baker and Wüstholz [1] and Waldschmidt [29]). Recent explicit upper bounds for the solutions of Thue equations have been given by Bugeaud and Györy [3]. Algorithms for the solution of a single Thue equation have been developed by several authors (see Bilu and Hanrot [2]).

E. Thomas [25] was the first to deal with a parametrized family of Thue equations; since then, some families have been investigated: cubic families have been discussed by Mignotte [16], Lee [12], and Mignotte and Tzanakis [19], a cubic inequality has been solved by Mignotte, Pethő, and Lemmermeyer [17]; quartic families have been considered by Pethő [20], Mignotte, Pethő, and Roth [18], Lettl and Pethő [13], Chen and Voutier [4], and Heuberger, Pethö, and Tichy [10]; Wakabayashi [28] dealt with a quartic inequality, Pethő and Tichy [21] solved a two-parametric quartic family, quintic families have been investigated by Heuberger [9] and Gaál and Lettl [6], a sextic family has been solved by Lettl, Pethő, and Voutier [14, 15]; see

1991 Mathematics Subject Classification: 11D41, 11D57.

This work was supported by Austrian National Bank project no. 7088 and the Austrian-Hungarian Science Cooperation project. 
also the overview in [10]. Most of these families have been solved by Baker's method using linear forms in logarithms, the others $[4,28,14]$ have been solved applying hypergeometric methods of Thue and Siegel.

A conjecture involving families of arbitrary degree has been made by E. Thomas [26]:

Conjecture 1. Let $n \geq 3$ and $p_{i} \in \mathbb{Z}[a]$ be monic polynomials for $2 \leq i \leq n$ such that

$$
0<\operatorname{deg} p_{2}<\operatorname{deg} p_{3}<\ldots<\operatorname{deg} p_{n} .
$$

Then there is a constant $a_{0}$ such that the Diophantine equation

$$
X \prod_{i=2}^{n}\left(X-p_{i}(a) Y\right)-Y^{n}= \pm 1
$$

has only solutions with $|y| \leq 1$ for all integers $a \geq a_{0}$.

Thomas [26] proved this conjecture for $n=3$ under some technical hypothesis.

Halter-Koch, Lettl, Pethő and Tichy [7] considered the family

$$
X\left(X-d_{2} Y\right) \ldots\left(X-d_{n-1} Y\right)(X-a Y) \pm Y^{n}= \pm 1
$$

for distinct integers $d_{i}$ and a parameter $a$. Based on a conjecture of Lang and Waldschmidt [11] they proved that there exists an effective constant $a_{0}$ such that for all $a \geq a_{0}$ the equation has only solutions with $|y| \leq 1$, provided that the corresponding number field is primitive, which is the case for almost all choices (in the sense of thin sets) of the parameters.

In Heuberger [8], the family

$$
(X+a Y)\left(X-d_{2} Y\right) \ldots\left(X-d_{n-1} Y\right)(X-a Y)-Y^{n}= \pm 1
$$

has been investigated for $n \geq 4$ and distinct integers $d_{2}, \ldots, d_{n-1}$. Also in this case, there is an effective constant $a_{0}$ such that for all $a \geq a_{0}$ the only solutions are those with $|y| \leq 1$, provided that $\sum d_{i} \neq 0$ or $\prod d_{i} \neq 0$.

In this paper we will present a similar result in the multi-parametrized case. We will use the following notations: For $p \in \mathbb{Z}\left[A_{1}, \ldots, A_{r}\right]$ we denote its homogeneous part of degree $k$ by $\mathrm{H}(p, k)$ and the homogeneous part of maximal degree by $\mathrm{LH}(p):=\mathrm{H}(p, \operatorname{deg} p)$.

Theorem 2. Let $n \geq 4, r \geq 1$ and $p_{i} \in \mathbb{Z}\left[A_{1}, \ldots, A_{r}\right]$ for $1 \leq i \leq n$. We make the following assumptions on the polynomials $p_{i}$ :

$$
\begin{gathered}
\operatorname{deg} p_{1}<\ldots<\operatorname{deg} p_{n-2}<\operatorname{deg} p_{n-1}=\operatorname{deg} p_{n}, \\
\operatorname{LH}\left(p_{n}\right)=\operatorname{LH}\left(p_{n-1}\right), \quad \text { but } p_{n} \neq p_{n-1} .
\end{gathered}
$$

Furthermore we suppose that for $p \in\left\{p_{1}, \ldots, p_{n}, p_{n}-p_{n-1}\right\}$, there exist 
positive constants $t_{p}, c_{p}$ such that

$$
\left|(\operatorname{LH}(p))\left(a_{1}, \ldots, a_{r}\right)\right| \geq c_{p} \cdot\left(\min _{k} a_{k}\right)^{\operatorname{deg} p} \quad \text { for } a_{1}, \ldots, a_{r} \geq t_{p} .
$$

We fix some $\tau \in \mathbb{R}$ satisfying

$$
\begin{aligned}
1<\tau< & 1+\min \left\{\frac{1}{\operatorname{deg} p_{n}-1},\right. \\
& \left.\frac{\operatorname{deg} p_{n}-\operatorname{deg}\left(p_{n}-p_{n-1}\right)}{\operatorname{deg} p_{n}+\operatorname{deg}\left(p_{n}-p_{n-1}\right)+\operatorname{deg} p_{n-2}}, \frac{\operatorname{deg} p_{n-2}-\operatorname{deg} p_{n-3}}{\operatorname{deg} p_{n}+\operatorname{deg} p_{n-3}}\right\} .
\end{aligned}
$$

Let

$$
F_{a_{1}, \ldots, a_{r}}(X, Y):=\prod_{i=1}^{n}\left(X-p_{i}\left(a_{1}, \ldots, a_{r}\right) Y\right)-Y^{n} .
$$

Then there is a constant $t_{0}$ such that for all $a_{1}, \ldots, a_{r}$ satisfying $t_{0} \leq \min _{k} a_{k}$ and

$$
\max _{k} a_{k} \leq\left(\min _{k} a_{k}\right)^{\tau}
$$

the Diophantine equation

$$
F_{a_{1}, \ldots, a_{r}}(x, y)= \pm 1
$$

considered for $x, y \in \mathbb{Z}$ has only the solutions

$$
\{( \pm 1,0)\} \cup\left\{ \pm\left(p_{i}\left(a_{1}, \ldots, a_{r}\right), 1\right): 1 \leq i \leq n\right\} .
$$

We note that (1) implies $\operatorname{deg} p_{n} \geq 2$ and $\operatorname{deg}\left(p_{n}-p_{n-1}\right)<\operatorname{deg} p_{n}$, therefore we are always able to fix a $\tau$ as described in (3).

In the case of one parameter only, we get the following corollary, writing $\operatorname{LT}(p)$ for the leading term of a univariate polynomial $p$.

Corollary 3. Let $n \geq 4$ and $p_{i} \in \mathbb{Z}[A]$ for $1 \leq i \leq n$. If

$$
\begin{gathered}
\operatorname{deg} p_{1}<\ldots<\operatorname{deg} p_{n-2}<\operatorname{deg} p_{n-1}=\operatorname{deg} p_{n}, \\
\operatorname{LT}\left(p_{n}\right)=\operatorname{LT}\left(p_{n-1}\right), \quad \text { but } p_{n} \neq p_{n-1},
\end{gathered}
$$

then there is a constant $t_{0}$ such that for all integers $a \geq t_{0}$ the Diophantine equation

$$
\prod_{i=1}^{n}\left(X-p_{i}(a) Y\right)-Y^{n}= \pm 1
$$

has only the solutions $\{( \pm 1,0)\} \cup\left\{ \pm\left(p_{i}(a), 1\right): 1 \leq i \leq n\right\}$.

The proof is based on Baker's method, more precisely, for estimating the large solutions we will apply a theorem of Bugeaud and Győry.

In Section 2 some asymptotic properties of various quantities are established for later use. Section 3 is devoted to properties of the associated number field, especially on estimates for the regulator. In Section 4 we will deal with approximation properties of solutions $(x, y)$ of $(5)$ and will find all 
solutions with very small $|y|$. Section 5 presents the crucial part of the proof: small solutions are excluded by subtle investigation of the asymptotics for the roots. The final Section 6 excludes large solutions applying the theorem of Bugeaud and Győry.

2. Asymptotic estimates. Throughout this paper, many arguments will be of asymptotic nature. In this section, we provide some asymptotic estimates which will be useful later.

In order to simplify notation, we write $t:=\min _{k} a_{k}$. For $1 \leq i \neq j \leq n$ we write $p_{i j}:=p_{i}-p_{j}, d_{i j}:=\operatorname{deg} p_{i j}$, and $d_{i}:=\operatorname{deg} p_{i}$.

We will use the usual $O_{-}, \Omega$ - and $\Theta$-notation for $t \rightarrow \infty$, where the implicit constants depend on $p_{1}, \ldots, p_{n}$. Sometimes, we will also use the notation $g \ll h$, where the implicit constants again depend on the given polynomials.

We note that (2), (1), and (4) imply for $1 \leq i \neq j \leq n$ and an arbitrary $p \in \mathbb{Z}\left[A_{1}, \ldots, A_{r}\right]$,

(6) $\quad t^{d_{i j}} \ll\left|\left(\operatorname{LH}\left(p_{i j}\right)\right)\left(a_{1}, \ldots, a_{r}\right)\right|$ and $\left|p\left(a_{1}, \ldots, a_{r}\right)\right| \ll t^{\tau \operatorname{deg} p}$.

We will often write $p_{i j}$ instead of $p_{i j}\left(a_{1}, \ldots, a_{r}\right)$ in order to simplify notation.

We define

$$
\mu:=\min \left\{\frac{d_{i j}-\tau\left(d_{i j}-1\right)}{2}: 1 \leq i \neq j \leq n, d_{i j} \geq 2\right\},
$$

and observe that $\mu<1 / 2$. For all $i, j$ with $d_{i j} \geq 2$, the conditions (3) and (1) yield

$$
d_{i j}-\tau\left(d_{i j}-1\right)>d_{i j}-\left(1+\frac{1}{d_{n}-1}\right)\left(d_{i j}-1\right)=\frac{d_{n}-d_{i j}}{d_{n}-1} \geq 0,
$$

which implies $\mu>0$.

LEMma 4 . For $1 \leq i \neq j \leq n$ the estimate

$$
\left|\frac{p_{i j}-\mathrm{LH}\left(p_{i j}\right)}{\operatorname{LH}\left(p_{i j}\right)}\right|=O\left(\frac{1}{t^{2 \mu}}\right)
$$

holds. In particular, this implies $p_{i j}\left(a_{1}, \ldots, a_{r}\right) \neq 0$ for sufficiently large $t$.

Pro of. If $d_{i j}=0$ then $\operatorname{LH}\left(p_{i j}\right)=p_{i j}$, and (8) follows trivially. If $d_{i j}=1$ then $p_{i j}-\mathrm{LH}\left(p_{i j}\right)$ is a constant, which implies with (6) that

$$
\left|\frac{p_{i j}-\mathrm{LH}\left(p_{i j}\right)}{\operatorname{LH}\left(p_{i j}\right)}\right| \ll \frac{1}{t} \leq \frac{1}{t^{2 \mu}},
$$

since $\mu<1 / 2$. Finally, we have to consider the case $d_{i j} \geq 2$. From (6) and (7) we get

$$
\left|\frac{p_{i j}-\mathrm{LH}\left(p_{i j}\right)}{\operatorname{LH}\left(p_{i j}\right)}\right| \ll \frac{t^{\tau\left(d_{i j}-1\right)}}{t^{d_{i j}}} \leq \frac{1}{t^{2 \mu}},
$$


which proves (8). We remark that (8) and (6) imply

$$
\left|p_{i j}\right| \geq\left|\operatorname{LH}\left(p_{i j}\right)\right|-\left|p_{i j}-\operatorname{LH}\left(p_{i j}\right)\right| \geq \frac{1}{2}\left|\operatorname{LH}\left(p_{i j}\right)\right| \gg t^{d_{i j}},
$$

therefore we obtain $\left|p_{i j}\right|>0$ for sufficiently large $t$.

Consider now the polynomial

$$
f(X):=F(X, 1)=\prod_{i=1}^{n}\left(X-p_{i}\right)-1 .
$$

We will need approximations for its roots.

LEMMA 5. Let

$$
q_{i}:=\prod_{\substack{j=1 \\ j \neq i}}^{n} \operatorname{LH}\left(p_{i j}\right)
$$

Then the roots of $f$ are all real and can be estimated as

$$
\alpha^{(i)}=p_{i}+\frac{1}{q_{i}}+\frac{1}{q_{i}} O\left(\frac{1}{t^{\mu}}\right), \quad 1 \leq i \leq n .
$$

Proof. We set

$$
\alpha_{i, M}:=p_{i}+\frac{1}{q_{i}}+\frac{1}{q_{i}} \cdot \frac{M}{t^{\mu}},
$$

where $M$ is a constant to be chosen later.

For $j \neq i$, Lemma 4, (6), $\operatorname{deg} q_{i} \geq 2$ (which follows from (1)), and $\mu<1 / 2$ give

$$
\begin{aligned}
\alpha_{i, M}-p_{j} & =p_{i j}+\frac{1}{q_{i}}\left(1+\frac{M}{t^{\mu}}\right) \\
& =\operatorname{LH}\left(p_{i j}\right)\left(1+\frac{p_{i j}-\operatorname{LH}\left(p_{i j}\right)}{\operatorname{LH}\left(p_{i j}\right)}+\frac{1}{q_{i} \operatorname{LH}\left(p_{i j}\right)}\left(1+\frac{M}{t^{\mu}}\right)\right) \\
& =\operatorname{LH}\left(p_{i j}\right)\left(1+O\left(\frac{1}{t^{2 \mu}}\right)+O\left(\frac{1}{t^{\operatorname{deg} q_{i}+d_{i j}}}\right)\right) \\
& =\operatorname{LH}\left(p_{i j}\right)\left(1+O\left(\frac{1}{t^{2 \mu}}\right)\right) .
\end{aligned}
$$

We obtain, for sufficiently large $t$,

$$
\left|f\left(\alpha_{i, M}\right)-\frac{M}{t^{\mu}}\right|=\left|1+\frac{M}{t^{\mu}}\right|\left|\prod_{j \neq i}\left(1+O\left(\frac{1}{t^{2 \mu}}\right)\right)-1\right| \leq \frac{c_{1}}{t^{2 \mu}},
$$

where the constant $c_{1}$ depends on the coefficients of $p_{1}, \ldots, p_{n}$ only (and not on $M$ ).

This implies $f\left(\alpha_{i,-1}\right)<0$ and $f\left(\alpha_{i, 1}\right)>0$ for sufficiently large $t$. Therefore there is a zero of $f$ between $\alpha_{i,-1}$ and $\alpha_{i, 1}$, which proves the assertion. 
For $1 \leq i, j \leq n$ we define $\eta_{i}^{(j)}:=\alpha^{(j)}-p_{i}$. We will frequently need the following estimate:

Lemma 6. The following asymptotic expansions hold for $1 \leq i, j \leq n$ :

$$
\log \left|\eta_{i}^{(j)}\right|= \begin{cases}\log \left|\operatorname{LH}\left(p_{j i}\right)\right|+O\left(1 / t^{2 \mu}\right) & \text { if } i \neq j, \\ -\log \left|q_{i}\right|+O\left(1 / t^{2 \mu}\right) & \text { if } i=j .\end{cases}
$$

Additionally, for $1 \leq i, j \leq n$ we have

$$
1 \ll \log \left|\eta_{i}^{(j)}\right| \ll \log t
$$

if $\{i, j\} \neq\{n-1, n\}$, we even have

$$
\log t \ll \log \left|\eta_{i}^{(j)}\right| .
$$

Proof. The assertion for $i \neq j$ follows from Lemma 5, Lemma 4, and $\log (1+z) \sim z$. To estimate $\log \left|\eta_{i}^{(i)}\right|$, we note that $f\left(\alpha^{(i)}\right)=0$ implies

$$
\log \left|\eta_{i}^{(i)}\right|=-\sum_{\substack{j=1 \\ j \neq i}}^{n} \log \left|\eta_{j}^{(i)}\right| .
$$

Finally, (9) and (10) are consequences of (6) and (3).

The following lemma will be used several times:

Lemma 7. Let $1 \leq k \leq n-2$ and let

$$
B_{k}:=\left(\begin{array}{cccc}
-\log \left|q_{1}\right| & \log \left|\operatorname{LH}\left(p_{12}\right)\right| & \ldots & \log \left|\operatorname{LH}\left(p_{1 k}\right)\right| \\
\log \left|\operatorname{LH}\left(p_{21}\right)\right| & -\log \left|q_{2}\right| & \ldots & \log \left|\operatorname{LH}\left(p_{2 k}\right)\right| \\
\vdots & \vdots & \ddots & \vdots \\
\log \left|\operatorname{LH}\left(p_{k 1}\right)\right| & \log \left|\operatorname{LH}\left(p_{k 2}\right)\right| & \ldots & -\log \left|q_{k}\right|
\end{array}\right) .
$$

Then

$$
\operatorname{det} B_{k}=\Theta\left(\log ^{k} t\right)
$$

Proof. The upper bound $\operatorname{det} B_{k}=O\left(\log ^{k} t\right)$ follows from (9).

Applying Gershgorin's estimate and (10) (which can be used since $k \leq$ $n-2)$ we obtain for an eigenvalue $\lambda$ of $B_{k}$ and for some $1 \leq i \leq k$,

$$
\begin{aligned}
|\lambda| & \geq \log \left|q_{i}\right|-|\lambda+\log | q_{i}|| \geq \log \left|q_{i}\right|-\sum_{\substack{l=1 \\
l \neq i}}^{k}|\log | \operatorname{LH}\left(p_{i l}\right)|| \\
& \geq \sum_{l=k+1}^{n}|\log | \operatorname{LH}\left(p_{i l}\right)|| \geq \log \left|\operatorname{LH}\left(p_{i n}\right)\right| \gg \log t .
\end{aligned}
$$

This implies $\operatorname{det} B_{k}=\Omega\left(\log ^{k} t\right)$. 
3. Properties of the number field. Let $\alpha^{(i)}$ be a root of $f$ and $K:=$ $\mathbb{Q}\left(\alpha^{(i)}\right)$ the corresponding number field. We denote by $\mathfrak{O}:=\mathbb{Z}\left[\alpha^{(i)}\right]$ the order generated by this root. We will extensively work in its unit group $\mathfrak{O}^{\times}$, therefore we will investigate the structure of this group.

It is a well known fact (see for example Schur [24]) that polynomials of the type of $f$ are irreducible (see also [8]).

Lemma 8. Let $n \geq 1$ and $d_{1}, \ldots, d_{n}$ be pairwise distinct integers. Then

$$
f(X):=\left(X-d_{1}\right) \ldots\left(X-d_{n}\right)-1
$$

is irreducible over $\mathbb{Q}$.

Therefore, Lemma 4 implies that $f$ is irreducible for sufficiently large $t$, and so by Lemma $5, K$ is a totally real number field of degree $n$ and of unit rank $n-1$ over $\mathbb{Q}$.

We estimate the regulator $R_{\mathfrak{O}}$ of the order $\mathfrak{O}$ using the following estimate of Pohst [22] (see also [8]).

TheOREM 9 (Pohst). Let $K$ be a totally real algebraic number field of degree $n \geq 4$, and $\mathfrak{O}$ an order of $K$ with discriminant $d_{\mathfrak{O}}$. Let $R_{\mathfrak{O}}$ be the regulator of $\mathfrak{O}$. Then

$$
R_{\mathfrak{O}} \geq c_{2} \log d_{\mathfrak{O}}
$$

where $c_{2}=c_{2}(n)$ is a constant depending only on $n$.

In our situation, this yields

Corollary 10. We have the estimate

$$
R_{\mathfrak{O}}=\Omega(\log t) .
$$

Pr o o f. By using Lemma 5, Lemma 4, and (6) the discriminant $d_{\mathfrak{O}}$ can be estimated by

$$
\begin{aligned}
\left|d_{\mathfrak{O}}\right| & =\left|\prod_{i=1}^{n} \prod_{j \neq i}\left(\alpha^{(i)}-\alpha^{(j)}\right)\right|=\left|\prod_{i=1}^{n} \prod_{j \neq i} \operatorname{LH}\left(p_{i j}\right)\left(1+O\left(\frac{1}{t^{2 \mu}}\right)\right)\right| \\
& =\left|\prod_{i=1}^{n} q_{i}\right|\left(1+O\left(\frac{1}{t^{2 \mu}}\right)\right)=\Omega\left(t^{\sum_{i} \operatorname{deg} q_{i}}\right) .
\end{aligned}
$$

Applying the above theorem gives the estimate for the regulator.

The $\eta_{j}^{(i)}$ as defined in Section 2 are units in $\mathfrak{O}$ and we even show in the following proposition that $\left\{\eta_{j}^{(i)}: 1 \leq j \leq n-1\right\}$ is an independent system of units which generates a subgroup of $\mathfrak{O}^{\times}$of reasonable index.

Proposition 11. Denote the group of units generated by $\eta_{k}^{(i)}, k=$ $1, \ldots, n-1$, and -1 by

$$
G:=\left\langle-1, \eta_{1}^{(i)}, \ldots, \eta_{n-1}^{(i)}\right\rangle .
$$


Then the regulator $R_{G}$ can be estimated by

$$
R_{G}=O\left(\log ^{n-1} t\right)
$$

and the index $I:=\left[\mathfrak{O}^{\times}: G\right]$ can be bounded by

$$
I=O\left(\log ^{n-2} t\right) .
$$

Additionally, we obtain $R_{G} / I=\Omega(\log t)$.

Proof. (11) is clear from (9).

Pohst and Zassenhaus [23, p. 361] and Corollary 10 imply

$$
I=\left[\mathfrak{O}^{\times}: G\right]=\frac{R_{G}}{R_{\mathfrak{O}}} \ll \frac{\log ^{n-1} t}{\log t} \ll \log ^{n-2} t
$$

and

$$
\frac{R_{G}}{I}=\frac{R_{G}}{R_{G}} R_{\mathfrak{O}}=\Omega(\log t)
$$

4. Approximation properties of solutions. We will first look for "trivial" solutions of (5). If $y=0$, we have $x^{n}= \pm 1$, and so $( \pm 1,0)$ is a solution. If $y= \pm 1$, we have either

$$
\prod_{i=1}^{n}\left(x-p_{i} y\right)=0,
$$

which yields the solutions $\left\{ \pm\left(p_{i}\left(a_{1}, \ldots, a_{r}\right), 1\right): 1 \leq i \leq n\right\}$, or

$$
\prod_{i=1}^{n}\left(x-p_{i} y\right)= \pm 2 \text {. }
$$

If all factors are distinct - which can be assumed for sufficiently large $t$ by Lemma 4 - there are no solutions of this equation due to the prime factor decomposition of 2 , since we assumed $n \geq 4$.

In the remainder of the paper, we will assume that there exists some solution with $|y| \geq 2$, and aim for a contradiction if $t$ is large enough.

We follow the classical lines (cf. Gaál [5]) to establish approximation properties of solutions. We have

$$
F(X, Y)=Y^{n} f(X / Y)=\prod_{j=1}^{n}\left(X-\alpha^{(j)} Y\right)=\mathrm{N}_{K / \mathbb{Q}}\left(X-\alpha^{(i)} Y\right) .
$$

Hence if $(x, y) \in \mathbb{Z}^{2}$ is a solution of (5) we have $\mathrm{N}_{K / \mathbb{Q}}\left(x-\alpha^{(i)} y\right)= \pm 1$ and therefore $\beta^{(i)}:=x-\alpha^{(i)} y$ is a unit of $\mathfrak{O}$. By Proposition 11 there is a representation

$$
\left(\beta^{(i)}\right)^{I}= \pm\left(\eta_{1}^{(i)}\right)^{u_{1}} \ldots\left(\eta_{n-1}^{(i)}\right)^{u_{n-1}}
$$

with integers $I, u_{1}, \ldots, u_{n-1}$, where $0<I \ll \log ^{n-2} t$. 
We define the type $j$ of a solution $(x, y)$ to be such that

$$
\left|\beta^{(j)}\right|=\min _{i=1, \ldots, n}\left|\beta^{(i)}\right| .
$$

Lemma 12. Let $(x, y) \in \mathbb{Z}^{2}$ be a solution of (5) of type $j$. Then we have the estimate

$$
\log \left|\beta^{(i)}\right|=\log |y|+\log \left|\eta_{j}^{(i)}\right|+O\left(1 / t^{e_{j}}\right), \quad i \neq j,
$$

where $e_{j}:=\operatorname{deg} q_{j}$.

Proof. We have

$$
|y| \cdot\left|\alpha^{(i)}-\alpha^{(j)}\right| \leq\left|\beta^{(i)}\right|+\left|\beta^{(j)}\right| \leq 2\left|\beta^{(i)}\right|,
$$

and by using $\mathrm{N}_{K / \mathbb{Q}}\left(\beta^{(i)}\right)= \pm 1$, Lemma 5 , and (6) we get

$$
\left|\beta^{(j)}\right|=\frac{1}{\prod_{i \neq j}\left|\beta^{(i)}\right|} \leq \frac{2^{n-1}}{|y|^{n-1} \prod_{i \neq j}\left|\alpha^{(i)}-\alpha^{(j)}\right|} \ll \frac{1}{t^{e_{j}}} .
$$

Therefore, by Lemma 4 and (6) we obtain for $i \neq j$,

$$
\left|\frac{\beta^{(i)}}{y}\right|=\left|\frac{x}{y}-\alpha^{(j)}+\alpha^{(j)}-p_{j}+p_{j}-\alpha^{(i)}\right|=\left|\eta_{j}^{(i)}\right|\left(1+O\left(\frac{1}{t^{e_{j}}}\right)\right) .
$$

5. Stable growth and small solutions. The aim of this section is to exclude "small" solutions, i.e. solutions with $|y|$ smaller than the (usually huge) upper bounds coming from linear form estimates. We will prove that if $(x, y)$ is a solution with $|y| \geq 2$, then

$$
\log |y| \geq t^{\kappa}
$$

for some $\kappa>0$ and sufficiently large $t$.

Such a property is the crucial point in the investigation of families of Thue equations using Baker's method and has been called "stable growth" by E. Thomas [26]. In fact, he considered a similar lower bound for $U:=$ $\max _{i}\left|u_{i}\right|$, which is related to $\log |y|$ by (12) and Lemma 12 in such a way that $U \approx \log ^{n-2} t \log |y|$.

Proposition 13. We have

$$
\log |y|=\Omega\left(\frac{t^{2 \mu}}{\log ^{n-4} t}\right) .
$$

Proof. By taking logarithms of conjugates of (12), we get a system of linear equations in $u_{k} / I, k=1, \ldots, n-1$,

$$
\log \left|\beta^{(i)}\right|=\frac{u_{1}}{I} \log \left|\eta_{1}^{(i)}\right|+\ldots+\frac{u_{n-1}}{I} \log \left|\eta_{n-1}^{(i)}\right|, \quad i \neq j,
$$


and denote the associated determinant by $R$, which is (up to sign) the regulator $R_{G}$ estimated in (11).

We will prove (13) depending on the type $j$ of the solution.

Assume $1 \leq j \leq n-2$. Without loss of generality we may assume $j=1$ (we will not use $\operatorname{deg} p_{1}<\ldots<\operatorname{deg} p_{n-1}$, but only $\operatorname{deg} p_{i}<\operatorname{deg} p_{n-1}=$ $\operatorname{deg} p_{n}$ for $1 \leq i \leq n-2$ in this part of the proof). Solving (14) by Cramer's rule and using Lemma 12 and (9) result in

$$
\begin{aligned}
R \frac{u_{n-1}}{I}= & \left|\begin{array}{cccc}
\log \left|\eta_{1}^{(2)}\right| & \ldots & \log \left|\eta_{n-2}^{(2)}\right| & \log \left|\beta^{(2)}\right| \\
\vdots & \ddots & \vdots & \vdots \\
\log \left|\eta_{1}^{(n)}\right| & \ldots & \log \left|\eta_{n-2}^{(n)}\right| & \log \left|\beta^{(n)}\right|
\end{array}\right| \\
= & \underbrace{\left|\begin{array}{cccc}
\log \left|\eta_{1}^{(2)}\right| & \ldots & \log \left|\eta_{n-2}^{(2)}\right| & 1 \\
\vdots & \ddots & \vdots & \vdots \\
\log \left|\eta_{1}^{(n)}\right| & \ldots & \log \left|\eta_{n-2}^{(n)}\right| & 1
\end{array}\right|}_{=: M_{1}} \log |y| \\
& +\underbrace{\left|\begin{array}{cccc}
\log \left|\eta_{1}^{(2)}\right| & \ldots & \log \left|\eta_{n-2}^{(2)}\right| & \log \left|\eta_{1}^{(2)}\right| \\
\vdots & \ddots & \vdots & \vdots \\
\log \left|\eta_{1}^{(n)}\right| & \ldots & \log \left|\eta_{n-2}^{(n)}\right| & \log \left|\eta_{1}^{(n)}\right|
\end{array}\right|+O\left(\frac{\log ^{n-2} t}{t^{e_{1}}}\right)}_{=: R_{1}},
\end{aligned}
$$

where $|R|=R_{G}$ is the regulator investigated in Proposition 11.

Obviously, $R_{1}=O\left(t^{-e_{1}} \log ^{n-2} t\right)$. We estimate $M_{1}$ :

$$
M_{1}=\left|\begin{array}{cccc}
\log \left|\eta_{1}^{(2)}\right| & \ldots & \log \left|\eta_{n-2}^{(2)}\right| & 1 \\
\vdots & \ddots & \vdots & \vdots \\
\log \left|\eta_{1}^{(n-1)}\right| & \ldots & \log \left|\eta_{n-2}^{(n-1)}\right| & 1 \\
\log \left|\eta_{1}^{(n)}\right|-\log \left|\eta_{1}^{(n-1)}\right| & \ldots & \log \left|\eta_{n-2}^{(n)}\right|-\log \left|\eta_{n-2}^{(n-1)}\right| & 0
\end{array}\right| .
$$

Note that (1) (especially $\operatorname{LH}\left(p_{n}\right)=\operatorname{LH}\left(p_{n-1}\right)$ ), Lemma 5, Lemma 4, (6), and $\log (1+z) \sim z$ imply for $1 \leq i \leq n-2$,

$$
\begin{aligned}
\log \left|\eta_{i}^{(n)}\right|-\log \left|\eta_{i}^{(n-1)}\right| & =\log \left|\frac{\alpha^{(n)}-p_{i}}{\alpha^{(n-1)}-p_{i}}\right|=\log \left(1+\frac{\alpha^{(n)}-\alpha^{(n-1)}}{\alpha^{(n-1)}-p_{i}}\right) \\
& =\frac{\operatorname{LH}\left(p_{n, n-1}\right)}{\operatorname{LH}\left(p_{n}\right)}\left(1+O\left(\frac{1}{t^{2 \mu}}\right)\right)=O\left(\frac{1}{t^{2 \mu}}\right),
\end{aligned}
$$

since $d_{n}-\tau d_{n, n-1} \geq d_{n, 1}-\tau\left(d_{n, 1}-1\right) \geq 2 \mu$ by (1) and (7). 
By Lemma 6 and (1) this yields

$$
\begin{aligned}
M_{1}= & \frac{\operatorname{LH}\left(p_{n, n-1}\right)}{\operatorname{LH}\left(p_{n}\right)} \\
& \times\left|\begin{array}{ccccc}
\log \left|\mathrm{LH}\left(p_{21}\right)\right| & -\log \left|q_{2}\right| & \ldots & \log \left|\mathrm{LH}\left(p_{2, n-2}\right)\right| & 1 \\
\vdots & \vdots & \ddots & \vdots & \vdots \\
\log \left|\mathrm{LH}\left(p_{n-2,1}\right)\right| & \log \left|\mathrm{LH}\left(p_{n-2,2}\right)\right| & \ldots & -\log \left|q_{n-2}\right| & 1 \\
\log \left|\mathrm{LH}\left(p_{n-1}\right)\right| & \log \left|\operatorname{LH}\left(p_{n-1}\right)\right| & \ldots & \log \left|\operatorname{LH}\left(p_{n-1}\right)\right| & 1 \\
1 & 1 & \ldots & 1 & 0
\end{array}\right| \\
& \times\left(1+O\left(\frac{\log ^{n-2} t}{t^{2 \mu}}\right)\right) .
\end{aligned}
$$

By subtracting $\log \left|\mathrm{LH}\left(p_{n-1}\right)\right|$ times the last row from the $(n-2)$ th row, expanding the determinant according to the $(n-2)$ th row and multiplying the last row by $2 \log \left|\mathrm{LH}\left(p_{n-1}\right)\right|$ we obtain

$$
\begin{aligned}
M_{1}= & -\frac{\operatorname{LH}\left(p_{n, n-1}\right)}{\operatorname{LH}\left(p_{n}\right)} \cdot \frac{1}{2 \log \left|\operatorname{LH}\left(p_{n-1}\right)\right|} \\
& \times\left|\begin{array}{cccc}
\log \left|\operatorname{LH}\left(p_{21}\right)\right| & -\log \left|q_{2}\right| & \ldots & \log \left|\operatorname{LH}\left(p_{2, n-2}\right)\right| \\
\vdots & \vdots & \ddots & \vdots \\
\log \left|\operatorname{LH}\left(p_{n-2,1}\right)\right| & \log \left|\operatorname{LH}\left(p_{n-2,2}\right)\right| & \ldots & -\log \left|q_{n-2}\right| \\
2 \log \left|\operatorname{LH}\left(p_{n-1}\right)\right| & 2 \log \left|\operatorname{LH}\left(p_{n-1}\right)\right| & \ldots & 2 \log \left|\operatorname{LH}\left(p_{n-1}\right)\right|
\end{array}\right| \\
& \times\left(1+O\left(\frac{\log ^{n-2} t}{t^{2 \mu}}\right)\right) .
\end{aligned}
$$

Summing up all rows in the last row, multiplying the last row by -1 and shifting the last row to the first row gives

$$
\begin{aligned}
M_{1}= & (-1)^{n+1} \frac{\mathrm{LH}\left(p_{n, n-1}\right)}{\operatorname{LH}\left(p_{n}\right)} \cdot \frac{1}{2 \log \left|\mathrm{LH}\left(p_{n-1}\right)\right|} \\
& \times\left|\begin{array}{cccc}
-\log \left|q_{1}\right| & \log \left|\operatorname{LH}\left(p_{12}\right)\right| & \ldots & \log \left|\operatorname{LH}\left(p_{1, n-2}\right)\right| \\
\log \left|\operatorname{LH}\left(p_{21}\right)\right| & -\log \left|q_{2}\right| & \ldots & \log \left|\operatorname{LH}\left(p_{2, n-2}\right)\right| \\
\vdots & \vdots & \ddots & \vdots \\
\log \left|\operatorname{LH}\left(p_{n-2,1}\right)\right| & \log \left|\operatorname{LH}\left(p_{n-2,2}\right)\right| & \ldots & -\log \left|q_{n-2}\right|
\end{array}\right| \\
& \times\left(1+O\left(\frac{\log ^{n-2} t}{t^{2 \mu}}\right)\right) .
\end{aligned}
$$

By estimating this last determinant using Lemma 7 for $k=n-2$ and (6), we get

$$
\frac{\log ^{n-3} t}{t^{\tau d_{n}-d_{n, n-1}}} \ll M_{1} \ll \frac{\log ^{n-3} t}{t^{2 \mu}} .
$$

Since $\tau d_{n}-d_{n, n-1}<e_{1}$ and since we assumed $|y| \geq 2$, (15) yields $R u_{n-1} / I \neq 0$. 
Therefore, the absolute value of the integer $u_{n-1}$ is at least one, which implies by (15), Proposition 11, and (16),

$$
\log |y| \geq \frac{1}{\left|M_{1}\right|}\left(\left|u_{n-1}\right|\left|\frac{R}{I}\right|-O\left(\frac{\log ^{n-2} t}{t^{e_{1}}}\right)\right) \gg \frac{\log t}{\left|M_{1}\right|} \gg \frac{t^{2 \mu}}{\log ^{n-4} t} .
$$

Assume now $n-1 \leq j \leq n$. Without loss of generality, we can assume $j=n$. Using (14) and Lemma 12 results in

$$
\begin{aligned}
R & \frac{u_{n-2}-u_{n-3}}{I} \\
= & \mid \begin{array}{ccccc}
\log \left|\eta_{1}^{(1)}\right| & \ldots & \log \left|\eta_{n-3}^{(1)}\right|+\log \left|\eta_{n-2}^{(1)}\right| & \log \left|\beta^{(1)}\right| & \log \left|\eta_{n-1}^{(1)}\right| \\
\vdots & \ddots & \vdots & \vdots & \vdots \\
\log \left|\eta_{1}^{(n-1)}\right| & \ldots & \log \left|\eta_{n-3}^{(n-1)}\right|+\log \left|\eta_{n-2}^{(n-1)}\right| & \log \left|\beta^{(n-1)}\right| & \log \left|\eta_{n-1}^{(n-1)}\right| \mid \\
= & \underbrace{\left|\begin{array}{ccccc}
\log \left|\eta_{1}^{(1)}\right| & \ldots & \log \left|\eta_{n-3}^{(1)}\right|+\log \left|\eta_{n-2}^{(1)}\right| & 1 & \log \left|\eta_{n-1}^{(1)}\right| \\
\vdots & \ddots & \vdots & \vdots & \vdots \\
\log \left|\eta_{1}^{(n-1)}\right| & \ldots & \log \left|\eta_{n-3}^{(n-1)}\right|+\log \left|\eta_{n-2}^{(n-1)}\right| & 1 & \log \left|\eta_{n-1}^{(n-1)}\right|
\end{array}\right|}_{=: M_{n}} \log |y|
\end{array} \\
& +R_{n},
\end{aligned}
$$

where

$$
\begin{aligned}
R_{n}= & \left|\begin{array}{ccccc}
\log \left|\eta_{1}^{(1)}\right| & \ldots & \log \left|\eta_{n-3}^{(1)}\right|+\log \left|\eta_{n-2}^{(1)}\right| & \log \left|\eta_{n}^{(1)}\right| & \log \left|\eta_{n-1}^{(1)}\right| \\
\vdots & \ddots & \vdots & \vdots & \vdots \\
\log \left|\eta_{1}^{(n-1)}\right| & \ldots & \log \left|\eta_{n-3}^{(n-1)}\right|+\log \left|\eta_{n-2}^{(n-1)}\right| & \log \left|\eta_{n}^{(n-1)}\right| & \log \left|\eta_{n-1}^{(n-1)}\right|
\end{array}\right| \\
& +O\left(\frac{\log ^{n-2} t}{t^{e_{n}}}\right) .
\end{aligned}
$$

Again, we have $R_{n}=O\left(\left(\log ^{n-2} t\right) / t^{e_{n}}\right)$ because the sum of the columns of the above matrix vanishes. We estimate $M_{n}$. Since $\sum_{k \neq n} \log \left|\eta_{k}^{(i)}\right|=$ $-\log \left|\eta_{n}^{(i)}\right|$, we obtain by column operations

$$
\begin{aligned}
M_{n} & =\left|\left(\log \left|\eta_{1}^{(i)}\right| \ldots \log \left|\eta_{n-4}^{(i)}\right|-\log \left|\eta_{n}^{(i)}\right| \quad 1 \quad \log \left|\eta_{n-1}^{(i)}\right|\right)_{1 \leq i \leq n-1}\right| \\
& =\mid\left(\log \left|\eta_{1}^{(i)}\right| \ldots \log \left|\eta_{n}^{(1)}\right|-\log \left|\eta_{n}^{(i)}\right|+\log \left|\eta_{n-1}^{(i)}\right|-\log \left|\eta_{n-1}^{(1)}\right|\right. \\
1 & \left.\log \left|\eta_{n-1}^{(i)}\right|\right)_{1 \leq i \leq n-1} \mid .
\end{aligned}
$$

We note that in the case of $n=4$, only the last three columns occur in the above matrix. 
Lemma 5 and (6) give for $1 \leq i \leq n-2$,

$$
\begin{aligned}
\log \left|\eta_{n}^{(1)}\right|-\log \left|\eta_{n}^{(i)}\right|+\log \left|\eta_{n-1}^{(i)}\right| & -\log \left|\eta_{n-1}^{(1)}\right| \\
= & \log \left(1+\frac{\left(p_{n}-p_{n-1}\right)\left(\alpha^{(1)}-\alpha^{(i)}\right)}{\left(p_{n}-\alpha^{(i)}\right)\left(p_{n-1}-\alpha^{(1)}\right)}\right) \\
= & O\left(\frac{1}{t^{2 d_{n}-\tau d_{n, n-1}-\tau d_{i, 1}}}\right)=O\left(\frac{1}{t^{\kappa_{3}}}\right),
\end{aligned}
$$

where $\kappa_{3}:=2 d_{n}-\tau d_{n, n-1}-\tau d_{n-2}$. Since $\log \left|\eta_{n}^{(1)}\right|-\log \left|\eta_{n}^{(n-1)}\right|+\log \left|\eta_{n-1}^{(n-1)}\right|$ $-\log \left|\eta_{n-1}^{(1)}\right|=\Theta(\log t)$ by Lemma 6 , expanding $M_{n}$ according to the $(n-3)$ th column yields

$$
\begin{aligned}
M_{n}= & \Theta(\log t)\left|\left(\log \left|\eta_{1}^{(i)}\right| \ldots \log \left|\eta_{n-4}^{(i)}\right| \quad 1 \quad \log \left|\eta_{n-1}^{(i)}\right|\right)_{1 \leq i \leq n-2}\right| \\
& +O\left(\frac{\log ^{n-3} t}{t^{\kappa_{3}}}\right) .
\end{aligned}
$$

By column operations we get

(17) $M_{n}=\Theta(\log t)$

$$
\begin{aligned}
& \times\left|\left(\log \left|\eta_{1}^{(i)}\right| \ldots \log \left|\eta_{n-4}^{(i)}\right| \quad 1 \log \left|\eta_{n-1}^{(i)}\right|-\log \left|\eta_{n-1}^{(1)}\right|\right)_{1 \leq i \leq n-2}\right| \\
& +O\left(\frac{\log ^{n-3} t}{t^{\kappa_{3}}}\right) .
\end{aligned}
$$

For $1 \leq i \leq n-3$ we obtain

$\log \left|\eta_{n-1}^{(i)}\right|-\log \left|\eta_{n-1}^{(1)}\right|=\log \left(1-\frac{\alpha^{(i)}-\alpha^{(1)}}{p_{n-1}-\alpha^{(1)}}\right)=O\left(\frac{1}{t^{d_{n}-\tau d_{i}}}\right)=O\left(\frac{1}{t^{\kappa_{2}}}\right)$,

where $\kappa_{2}:=d_{n}-\tau d_{n-3}$. Setting $\kappa_{1}:=\tau d_{n}-d_{n-2}$ gives

$$
\begin{aligned}
\frac{1}{t^{\kappa_{1}}} & \ll \log \left|\eta_{n-1}^{(n-2)}\right|-\log \left|\eta_{n-1}^{(1)}\right|=\log \left(1-\frac{\alpha^{(n-2)}-\alpha^{(1)}}{p_{n-1}-\alpha^{(1)}}\right) \\
& \ll \frac{1}{t^{d_{n-1}-\tau d_{n-2}}} .
\end{aligned}
$$

We note that (3) implies

$$
\kappa_{1}<\min \left(\kappa_{2}, \kappa_{3}\right)=: \kappa_{4}
$$

Expanding the determinant in (17) according to the last column yields

$$
\begin{aligned}
M_{n}= & \Theta(\log t) \log \left|\frac{\eta_{n-1}^{(n-2)}}{\eta_{n-1}^{(1)}}\right| \cdot\left|\left(\log \left|\eta_{1}^{(i)}\right| \ldots \log \left|\eta_{n-4}^{(i)}\right| 1\right)_{1 \leq i \leq n-3}\right| \\
& +O\left(\frac{\log ^{n-3} t}{t^{\kappa_{4}}}\right) .
\end{aligned}
$$


By Lemma 6 we have

$$
\begin{aligned}
M_{n}= & \Theta(\log t) \log \left|\frac{\eta_{n-1}^{(n-2)}}{\eta_{n-1}^{(1)}}\right|\left(M_{n}^{\prime}+O\left(\frac{\log ^{n-5} t}{t^{2 \mu}}\right)\right) \\
& +O\left(\frac{\log ^{n-3} t}{t^{\kappa_{4}}}\right)
\end{aligned}
$$

where

$$
M_{n}^{\prime}=\left|\begin{array}{cccc}
-\log \left|q_{1}\right| & \ldots & \log \left|\operatorname{LH}\left(p_{1, n-4}\right)\right| & 1 \\
\vdots & \ddots & \vdots & \vdots \\
\log \left|\operatorname{LH}\left(p_{n-4,1}\right)\right| & \ldots & -\log \left|q_{n-4}\right| & 1 \\
\log \left|\operatorname{LH}\left(p_{n-3,1}\right)\right| & \ldots & \log \left|\operatorname{LH}\left(p_{n-3, n-4}\right)\right| & 1
\end{array}\right| .
$$

We note that from (1) it follows for $1 \leq i \leq n-3$ that

$$
\begin{aligned}
\ell_{i} & :=\log \left|\operatorname{LH}\left(p_{i, n-2}\right)\right|+\log \left|\operatorname{LH}\left(p_{i, n-1}\right)\right|+\log \left|\operatorname{LH}\left(p_{i, n}\right)\right| \\
& =\log \left|\operatorname{LH}\left(p_{n-2}\right)\right|+\log \left|\operatorname{LH}\left(p_{n-1}\right)\right|+\log \left|\operatorname{LH}\left(p_{n}\right)\right|
\end{aligned}
$$

is independent of $i$, therefore we obtain

$$
\begin{aligned}
& M_{n}^{\prime}=\frac{1}{\log \left|\operatorname{LH}\left(p_{n-2}\right)\right|+\log \left|\operatorname{LH}\left(p_{n-1}\right)\right|+\log \left|\operatorname{LH}\left(p_{n}\right)\right|} \\
& \times\left|\begin{array}{cccc}
-\log \left|q_{1}\right| & \ldots & \log \left|\operatorname{LH}\left(p_{1, n-4}\right)\right| & \ell_{1} \\
\vdots & \ddots & \vdots & \vdots \\
\log \left|\operatorname{LH}\left(p_{n-4,1}\right)\right| & \ldots & -\log \left|q_{n-4}\right| & \ell_{n-4} \\
\log \left|\operatorname{LH}\left(p_{n-3,1}\right)\right| & \ldots & \log \left|\operatorname{LH}\left(p_{n-3, n-4}\right)\right| & \ell_{n-3}
\end{array}\right| \\
& =\Theta\left(\frac{1}{\log t}\right) \\
& \times\left|\begin{array}{cccc}
-\log \left|q_{1}\right| & \ldots & \log \left|\operatorname{LH}\left(p_{1, n-4}\right)\right| & \log \left|\operatorname{LH}\left(p_{1, n-3}\right)\right| \\
\vdots & \ddots & \vdots & \vdots \\
\log \left|\operatorname{LH}\left(p_{n-4,1}\right)\right| & \ldots & -\log \left|q_{n-4}\right| & \log \left|\operatorname{LH}\left(p_{n-4, n-3}\right)\right| \\
\log \left|\operatorname{LH}\left(p_{n-3,1}\right)\right| & \ldots & \log \left|\operatorname{LH}\left(p_{n-3, n-4}\right)\right| & -\log \left|q_{n-3}\right|
\end{array}\right| \\
& =\Theta\left(\frac{1}{\log t}\right) \cdot \Theta\left(\log ^{n-3} t\right)
\end{aligned}
$$

by Lemma 7 . This implies, with (18) and (19) that

$$
\frac{\log ^{n-3} t}{t^{\tau d_{n}-d_{n-2}}} \ll M_{n} \ll \frac{\log ^{n-3} t}{t^{d_{n}-\tau d_{n-2}}} \ll \frac{\log ^{n-3} t}{t^{2 \mu}},
$$

since $d_{n}-\tau d_{n-2} \geq d_{n}-\tau\left(d_{n}-1\right) \geq 2 \mu$. Using the same argument as in the case $j=1$, we prove the asserted estimate for $\log |y|$. 
6. Large solutions. To exclude the possibility of "large" solutions, we use a result of Bugeaud and Győry.

Theorem 14 (Bugeaud-Györy [3]). Let $F \in \mathbb{Z}[X, Y]$ be a homogeneous irreducible polynomial of degree $n \geq 3$ and $0 \neq m \in \mathbb{Z}$. Let $B \geq \max \{|m|$, e\}, $\alpha$ a zero of $F(X, 1), K:=\mathbb{Q}(\alpha), R:=R_{K}$ the regulator and $r$ the unit rank of $K$. Let $H \geq 3$ be an upper bound for the absolute value of the coefficients of $F$. Then all solutions $(x, y) \in \mathbb{Z}^{2}$ of $F(x, y)=m$ satisfy

$$
\max \{|x|,|y|\}<\exp \left(c_{3} \cdot R \cdot \max \{\log R, 1\} \cdot(R+\log (H B))\right)
$$

and

$$
\max \{|x|,|y|\}<\exp \left(c_{4} \cdot H^{2 n-2} \cdot \log ^{2 n-1} H \cdot \log B\right),
$$

where

$c_{3}=c_{3}(n, r)=3^{r+27}(r+1)^{7 r+19} n^{2 n+6 r+14}, \quad c_{4}=c_{4}(n)=3^{3(n+9)} n^{18(n+1)}$.

This gives

$$
\log |y| \leq c_{3} R_{K} \log R_{K}\left(R_{K}+\log (H B)\right),
$$

where by Proposition 11 and (6),

$$
R_{K} \leq R_{G}=O\left(\log ^{n-1} t\right), \quad B=e, \quad \log H=O(\log t),
$$

and therefore $\log |y|=O\left(\log ^{2 n-1} t\right)$. This is a contradiction to (13) for large $t$ and thus Theorem 2 is proved.

REMARK. We note that $c_{3}$ is usually a rather big constant, therefore for the practical solution of a particular family it may be more advantageous to use directly a lower bound for linear forms in logarithms.

Acknowledgements. The authors are grateful to the referee for his careful reading of the manuscript and his suggestion to improve (4) to its present form.

\section{References}

[1] A. Baker and G. Wüstholz, Logarithmic forms and group varieties, J. Reine Angew. Math. 442 (1993), 19-62.

[2] Yu. Bilu and G. Hanrot, Solving Thue equations of high degree, J. Number Theory 60 (1996), 373-392.

[3] Y. Bugeaud and K. Győry, Bounds for the solutions of Thue-Mahler equations and norm form equations, Acta Arith. 74 (1996), 273-292.

[4] J. H. Chen and P. M. Voutier, Complete solution of the Diophantine equation $X^{2}+1=d Y^{4}$ and a related family of quartic Thue equations, J. Number Theory 62 (1997), 71-99. 
[5] I. Gaál, On the resolution of some Diophantine equations, in: Computational Number Theory, A. Pethő, M. Pohst, H. C. Williams and H. G. Zimmer (eds.), de Gruyter, Berlin, 1991, 261-280.

[6] I. Gaál and G. Lettl, A parametric family of quintic Thue equations, Math. Comp., to appear.

[7] F. Halter-Koch, G. Lettl, A. Pethő and R. F. Tichy, Thue equations associated with Ankeny-Brauer-Chowla number fields, J. London Math. Soc., to appear.

[8] C. Heuberger, On families of parametrized Thue equations, J. Number Theory 76 (1999), 45-61.

[9] -, On a family of quintic Thue equations, J. Symbolic Comput. 26 (1998), 173-185.

[10] C. Heuberger, A. Pethő and R. F. Tichy, Complete solution of parametrized Thue equations, Acta Math. Inform. Univ. Ostraviensis 6 (1998), 93-113.

[11] S. Lang, Elliptic Curves: Diophantine Analysis, Grundlehren Math. Wiss. 23, Springer, Berlin, 1978.

[12] E. Lee, Studies on Diophantine equations, Ph.D. thesis, Cambridge Univ., 1992.

[13] G. Lettl and A. Pethő, Complete solution of a family of quartic Thue equations, Abh. Math. Sem. Univ. Hamburg 65 (1995), 365-383.

[14] G. Lettl, A. Pethő and P. Voutier, Simple families of Thue inequalities, Trans. Amer. Math. Soc. 351 (1999), 1871-1894.

[15] - - - - On the arithmetic of simplest sextic fields and related Thue equations, in: Number Theory, Diophantine, Computational and Algebraic Aspects (Eger, 1996), K. Győry, A. Pethő and V. T. Sós (eds.), de Gruyter, Berlin, 1998, 331-348.

[16] M. Mignotte, Verification of a conjecture of E. Thomas, J. Number Theory 44 (1993), 172-177.

[17] M. Mignotte, A. Pethő and F. Lemmermeyer, On the family of Thue equations $x^{3}-(n-1) x^{2} y-(n+2) x y^{2}-y^{3}=k$, Acta Arith. 76 (1996), 245-269.

[18] M. Mignotte, A. Pethö and R. Roth, Complete solutions of quartic Thue and index form equations, Math. Comp. 65 (1996), 341-354.

[19] M. Mignotte and N. Tzanakis, On a family of cubics, J. Number Theory 39 (1991), 41-49.

[20] A. Pethő, Complete solutions to families of quartic Thue equations, Math. Comp. 57 (1991), 777-798.

[21] A. Pethő and R. F. Tichy, On two-parametric quartic families of Diophantine problems, J. Symbolic Comput. 26 (1998), 151-171.

[22] M. Pohst, Regulatorabschätzungen für total reelle algebraische Zahlkörper, J. Number Theory 9 (1977), 459-492.

[23] M. Pohst and H. Zassenhaus, Algorithmic Algebraic Number Theory, Cambridge Univ. Press, Cambridge, 1989.

[24] I. Schur, Aufgabe 226, Arch. Math. Physik 13 (1908), 367.

[25] E. Thomas, Complete solutions to a family of cubic Diophantine equations, J. Number Theory 34 (1990), 235-250.

[26] - Solutions to certain families of Thue equations, ibid. 43 (1993), 319-369.

[27] A. Thue, Über Annäherungswerte algebraischer Zahlen, J. Reine Angew. Math. 135 (1909), 284-305.

[28] I. Wakabayashi, On a family of quartic Thue inequalities I, J. Number Theory 66 (1997), 70-84. 
[29] M. Waldschmidt, Minoration de combinaisons linéaires de logarithmes de nombres algébriques, Canad. J. Math. 45 (1993), 176-224.

Institut für Mathematik

Technische Universität Graz

Steyrergasse 30

A-8010 Graz, Austria

E-mail: cheub@weyl.math.tu-graz.ac.at tichy@weyl.math.tu-graz.ac.at

Received on 13.11 .1998

and in revised form on 13.4.1999 